Mon. Not. R. Astron. Soc. 000,166() Printed 27 July $2021 \quad$ (MN LATEX style file v2.2)

\title{
On the magnetic field evolution timescale in superconducting neutron star cores
}

\author{
Andrea Passamonti`, Taner Akgün, José A. Pons, Juan A. Miralles \\ Departament de Física Aplicada, Universitat d'Alacant, Ap. Correus 99, 03080 Alacant, Spain
}

27 July 2021

\begin{abstract}
We revisit the various approximations employed to study the long-term evolution of the magnetic field in neutron star cores and discuss their limitations and possible improvements. A recent controversy on the correct form of the induction equation and the relevant evolution timescale in superconducting neutron star cores is addressed and clarified. We show that this ambiguity in the estimation of timescales arises as a consequence of nominally large terms that appear in the induction equation, but which are, in fact, mostly irrotational. This subtlety leads to a discrepancy by many orders of magnitude when velocity fields are absent or ignored. Even when internal velocity fields are accounted for, only the solenoidal part of the electric field contributes to the induction equation, which can be substantially smaller than the irrotational part. We also argue that stationary velocity fields must be incorporated in the slow evolution of the magnetic field as the next level of approximation.
\end{abstract}

Key words: stars: evolution - stars: magnetars - stars: magnetic field -stars: neutron.

\section{INTRODUCTION}

The evolution of the magnetic field in the interior of neutron stars is a complex and controversial research area, with several important open issues. Part of the difficulty stems from the multi-fluid character of the problem, with at least three different species (protons, neutrons, and electrons) that are not necessarily coupled in some range of temperatures and timescales of interest. This makes necessary a multi-fluid dynamics approach to the problem. In spite of recent relevant advances in the field, many important aspects are still under debate. For instance, we still ignore the 'equilibrium' configuration which emerges from the star formation after a core-collapse, and how the subsequent long-term evolution of the magnetic field in the core proceeds.

The complex multi-fluid physics becomes particularly difficult when protons/neutrons in the core undergo a transition to a superconducting/superfluid state, further decoupling the components. Recently, there has been a number or relevant papers aiming at improving the description and understanding of non trivial interactions between the various particle species. Glampedakis, Andersson \& Samuelsson (2011) derived the MHD equations for superfluid and type II superconducting neutron stars, by using a Newtonian variational formalism and clarifying several aspects of these systems. Their equations were consistent with those determined

\footnotetext{
* E-mail:passamonti@ua.es
}

by Mendell \& Lindblom (1991) and Mendell (1991a, b) , and they recognized the role of the London magnetic field in the superconducting equivalent to Ampér's law. This formalism has been later applied by Graber et al. (2015) and Elfritz et al. (2016) to study the induction equation of superconducting neutron stars and to estimate the (very long) timescales of the magnetic field evolution. More recently, Gusakov (2016) and Gusakov \& Dommes (2016) have derived and further extended the HVBK formalism originally derived to describe the dynamics of superfluid helium (Hall \& Vinen 1956; Hall 1960; Bekarevich \& Khalatnikov 1961; Khalatnikov 2000) to general relativity. This formalism allows to study superfluid and type I and type II superconducting neutron stars with thermal effects. The correct implementation of the buoyancy effect has also been discussed in Dommes \& Gusakov (2017).

Generally the various formalisms provide equivalent descriptions of the physical system, and they agree on the most part of the equations, but there are also discrepancies. The main controversy, which appeared recently, concerns the form of the induction equation in superconducting neutron stars. The electric field determined by Gusakov \& Dommes (2016) has an extra contribution of the fluxtube tension which is absent in the derivation of Glampedakis et al. (2011) and Graber et al. (2015). As further emphasized in Dommes \& Gusakov (2017), this leads to a difference of several orders of magnitude in the estimated evolution timescales. 
The paradox is that for type II superconducting stars the equations, which have been derived by these two groups, are completely equivalent with the only exception of the induction equation. This issue, as we will show later, is due to the different assumptions the authors have made to find the electric field, in particular, neglecting the inertial terms in the momentum and mass conservation equations to estimate the long-term timescales. In this paper we revisit these assumptions, discuss their limitations, and propose how to improve the current calculations and to reconcile apparently opposed results. We will proceed gradually, from the simplest case to the most complex, showing that the basic math and physics assumptions are analogous in a simple case and a complex superconducting liquid.

The paper is organized as follows. In Section 2 we describe the case of normal matter. We start by considering an electron-proton plasma, excluding neutrons for clarity, and then go on to discuss the more general case when neutrons are included as well. The superconducting case is treated in Section 3 Finally, Section 4 is dedicated to the concluding remarks.

\section{MAGNETIC FIELD EVOLUTION IN NORMAL MATTER}

\subsection{Magnetized two-component plasma}

We begin by studying the simplest case. Consider the dynamical equations for a two-fluid system composed of two charged components. These two components are denoted with the letters $\mathrm{p}$ (positively charged with charge $+e$ ) and e (negatively charged with charge $-e$ ). For simplicity, we also assume that each component individually obeys a barotropic equation of state. In addition to the number (and mass) conservation for each fluid,

$$
\frac{\partial n_{\mathrm{x}}}{\partial t}+\nabla \cdot\left(n_{\mathrm{x}} \boldsymbol{v}_{\mathrm{x}}\right)=0
$$

the Euler equations for the two species can be written in the following form,

$$
\begin{aligned}
\rho_{\mathrm{p}}\left(\frac{\partial \boldsymbol{v}_{\mathrm{p}}}{\partial t}+\boldsymbol{v}_{\mathrm{p}} \cdot \boldsymbol{\nabla} \boldsymbol{v}_{\mathrm{p}}\right) & +n_{\mathrm{p}} \boldsymbol{\nabla} \mu_{\mathrm{p}}+\rho_{\mathrm{p}} \boldsymbol{\nabla} \Phi= \\
& =\boldsymbol{F}_{\mathrm{pe}}+e n_{\mathrm{p}}\left(\boldsymbol{E}+\frac{\boldsymbol{v}_{\mathrm{p}}}{c} \times \boldsymbol{B}\right), \\
\rho_{\mathrm{e}}\left(\frac{\partial \boldsymbol{v}_{\mathrm{e}}}{\partial t}+\boldsymbol{v}_{\mathrm{e}} \cdot \boldsymbol{\nabla} \boldsymbol{v}_{\mathrm{e}}\right) & +n_{\mathrm{e}} \boldsymbol{\nabla} \mu_{\mathrm{e}}+\rho_{\mathrm{e}} \boldsymbol{\nabla} \Phi= \\
& =\boldsymbol{F}_{\mathrm{ep}}-e n_{\mathrm{e}}\left(\boldsymbol{E}+\frac{\boldsymbol{v}_{\mathrm{e}}}{c} \times \boldsymbol{B}\right) .
\end{aligned}
$$

Here $\rho_{\mathrm{x}}, n_{\mathrm{x}}, \mu_{\mathrm{x}}$, and $\boldsymbol{v}_{\mathrm{x}}$ are, respectively, the mass density, number density, chemical potential and velocity of the $\mathrm{x}$ fluid (x = e, p), $\boldsymbol{E}$ and $\boldsymbol{B}$ are the electric and magnetic fields measured in the laboratory frame, and $\Phi$ is the gravitational potential. The quantity $\boldsymbol{F}_{\mathrm{xy}}$ is a drag force between the two fluids of the form $\boldsymbol{F}_{\mathrm{xy}}=-\frac{m_{\mathrm{x}} n_{\mathrm{x}}}{\tau_{\mathrm{xy}}}\left(\boldsymbol{v}_{\mathrm{x}}-\boldsymbol{v}_{\mathrm{y}}\right)=-\boldsymbol{F}_{\mathrm{yx}}$, with $\tau_{\mathrm{xy}}$ being relaxation times (note that $\mathrm{x} \neq \mathrm{y}$ ).

Adding equations (2) and (3) one obtains an equation similar to the Euler equation for a magnetized fluid. Assuming local charge neutrality $\left(n_{\mathrm{p}}=n_{\mathrm{e}} \equiv n_{\mathrm{c}}\right)$ and dropping the inertial terms, this reduces to the magnetohydrostatic equilibrium equation

$$
n_{\mathrm{c}} \boldsymbol{\nabla}\left(\mu_{\mathrm{p}}+\mu_{\mathrm{e}}\right)+\rho_{\mathrm{c}} \boldsymbol{\nabla} \Phi=\boldsymbol{F}_{\mathrm{L}},
$$

where $\rho_{\mathrm{c}}=\rho_{\mathrm{p}}+\rho_{\mathrm{e}}=\left(m_{\mathrm{p}}+m_{\mathrm{e}}\right) n_{\mathrm{c}}$ is the total mass density of the charged fluid and the Lorentz force is given by

$$
\boldsymbol{F}_{\mathrm{L}}=\frac{\boldsymbol{j} \times \boldsymbol{B}}{c}
$$

The current $j$ is related to the relative velocity between the two charged fluids $\boldsymbol{u} \equiv \boldsymbol{v}_{\mathrm{p}}-\boldsymbol{v}_{\mathrm{e}}$ through $\boldsymbol{j}=e n_{\mathrm{c}} \boldsymbol{u}$. To close the system one ha to solve the Poisson equation to determine $\Phi$ and can specify the current in terms of the magnetic field by Ampère's law,

$$
\boldsymbol{j}=\frac{c}{4 \pi} \nabla \times \boldsymbol{B} .
$$

We can combine the chemical potentials and the gravitational terms in equation (4) into a single gradient, which then implies that the Lorentz force per unit charge, $\boldsymbol{F}_{\mathrm{L}} / n_{\mathrm{C}}$ must be the gradient of a scalar function. This requirement can be expressed as

$$
\nabla \times\left(\frac{\boldsymbol{F}_{\mathrm{L}}}{n_{\mathrm{c}}}\right)=0 .
$$

This is an important point to bear in mind when we consider the electric field from the dynamical equations below.

The evolution of the magnetic field is given by the induction equation,

$$
\frac{\partial \boldsymbol{B}}{\partial t}=-c \boldsymbol{\nabla} \times \boldsymbol{E}
$$

To study the long-term evolution of the magnetic field it is necessary to adopt some approximations in order to isolate the effects of the slow processes from the fast dynamics. A common practice is to neglect the inertial terms in writing the electric field from the Euler equations. This means that velocities are assumed to be small and to vary on much longer timescales than any of the relaxation times. In that limit, the electric field can be written from either of the two equations (2) and (3), as

$$
\begin{aligned}
& \left.\boldsymbol{E}\right|_{\mathrm{p}} \simeq-\frac{\boldsymbol{F}_{\mathrm{L}}}{e n_{\mathrm{c}}}-\frac{\boldsymbol{F}_{\mathrm{pe}}}{e n_{\mathrm{c}}}-\frac{\boldsymbol{v}_{\mathrm{e}}}{c} \times \boldsymbol{B}+\frac{1}{e} \boldsymbol{\nabla} \mu_{\mathrm{p}}+\frac{m_{\mathrm{p}}}{e} \nabla \Phi, \\
& \left.\boldsymbol{E}\right|_{\mathrm{e}} \simeq-\frac{\boldsymbol{F}_{\mathrm{pe}}}{e n_{\mathrm{c}}}-\frac{\boldsymbol{v}_{\mathrm{e}}}{c} \times \boldsymbol{B}-\frac{1}{e} \boldsymbol{\nabla} \mu_{\mathrm{e}}-\frac{m_{\mathrm{e}}}{e} \boldsymbol{\nabla} \Phi,
\end{aligned}
$$

where the label of the electric field specifies the Euler equation from which it has been derived. Here, the first equation is written in a way showing explicitly that the difference between the two expressions is the Lorentz force per unit charge and some gradient terms. Obviously, the two expressions are exactly equivalent if one uses the equilibrium equation (4). As noted above, however, this equilibrium equation additionally requires the Lorentz force per unit charge to be a gradient, imposing a severe restriction on the form of the magnetic field which is not guaranteed to be automatically satisfied as the magnetic field evolves. Thus, the two equations are equivalent for the induction equation only if the magnetic field further satisfies the restriction in equation (17).

More generally, when $\boldsymbol{F}_{\mathrm{L}} / n_{\mathrm{c}}$ has a solenoidal part in addition to the irrotational part, it no longer satisfies the static equilibrium equation (4), and velocity fields must be present. This point is occasionally overlooked. In this case, the approximate forms of $\left.\boldsymbol{E}\right|_{\mathrm{p}}$ and $\left.\boldsymbol{E}\right|_{\mathrm{e}}$ from the above equations will no longer lead to the same magnetic field evolution. In order to see which of the two approximations is better, 
we can write the exact vector $\boldsymbol{E}$ in the following two forms

$$
\begin{aligned}
& \boldsymbol{E}=\left.\boldsymbol{E}\right|_{\mathrm{p}}+\frac{m_{\mathrm{p}}}{e}\left(\frac{\partial \boldsymbol{v}_{\mathrm{p}}}{\partial t}+\boldsymbol{v}_{\mathrm{p}} \cdot \boldsymbol{\nabla} \boldsymbol{v}_{\mathrm{p}}\right), \\
& \boldsymbol{E}=\left.\boldsymbol{E}\right|_{\mathrm{e}}-\frac{m_{\mathrm{e}}}{e}\left(\frac{\partial \boldsymbol{v}_{\mathrm{e}}}{\partial t}+\boldsymbol{v}_{\mathrm{e}} \cdot \boldsymbol{\nabla} \boldsymbol{v}_{\mathrm{e}}\right),
\end{aligned}
$$

where we have re-introduced the inertial terms. We have two other constraints: (i) the mass carried by electrons is much smaller than that of the protons, $m_{\mathrm{e}} \ll m_{\mathrm{p}}$, and (ii) the two fluids have very similar velocities, $\boldsymbol{v}_{\mathrm{p}} \simeq \boldsymbol{v}_{\mathrm{e}}$, i.e. the macroscopic electric current is very small compared to the mass current because of the strong electromagnetic coupling between the opposed charges. If both conditions apply, it is more reasonable to assume that $\left.\boldsymbol{E}\right|_{\mathrm{e}}$ is a better approximation of the electric field, as the correction due to the inertial terms of the electrons is smaller.

\subsection{Generalized Ohm's law in neutron star matter}

More generally, let's consider the case when neutrons are also present and interact with protons and electrons. We now have equations of motion for three constituents, with their respective frictional couplings

$$
\begin{aligned}
\rho_{\mathrm{p}}\left(\frac{\partial \boldsymbol{v}_{\mathrm{p}}}{\partial t}+\boldsymbol{v}_{\mathrm{p}} \cdot \boldsymbol{\nabla} \boldsymbol{v}_{\mathrm{p}}\right) & +n_{\mathrm{p}} \boldsymbol{\nabla} \mu_{\mathrm{p}}+\rho_{\mathrm{p}} \boldsymbol{\nabla} \Phi= \\
= & e n_{\mathrm{p}}\left(\boldsymbol{E}+\frac{\boldsymbol{v}_{\mathrm{p}}}{c} \times \boldsymbol{B}\right)+\boldsymbol{F}_{\mathrm{pe}}+\boldsymbol{F}_{\mathrm{pn}},
\end{aligned}
$$

$$
\begin{aligned}
\rho_{\mathrm{e}}\left(\frac{\partial \boldsymbol{v}_{\mathrm{e}}}{\partial t}+\boldsymbol{v}_{\mathrm{e}} \cdot \boldsymbol{\nabla} \boldsymbol{v}_{\mathrm{e}}\right) & +n_{\mathrm{e}} \boldsymbol{\nabla} \mu_{\mathrm{e}}+\rho_{\mathrm{e}} \boldsymbol{\nabla} \Phi= \\
= & -e n_{\mathrm{e}}\left(\boldsymbol{E}+\frac{\boldsymbol{v}_{\mathrm{e}}}{c} \times \boldsymbol{B}\right)+\boldsymbol{F}_{\mathrm{ep}}+\boldsymbol{F}_{\mathrm{en}}
\end{aligned}
$$

$\rho_{\mathrm{n}}\left(\frac{\partial \boldsymbol{v}_{\mathrm{n}}}{\partial t}+\boldsymbol{v}_{\mathrm{n}} \cdot \boldsymbol{\nabla} \boldsymbol{v}_{\mathrm{n}}\right)+n_{\mathrm{n}} \boldsymbol{\nabla} \mu_{\mathrm{n}}+\rho_{\mathrm{n}} \boldsymbol{\nabla} \Phi=\boldsymbol{F}_{\mathrm{np}}+\boldsymbol{F}_{\mathrm{ne}}$.

Adding the three equations, assuming local charge neutrality, and dropping the inertial terms, one obtains the global static equilibrium equation

$$
n_{\mathrm{c}} \boldsymbol{\nabla}\left(\mu_{\mathrm{p}}+\mu_{\mathrm{e}}\right)+n_{\mathrm{n}} \boldsymbol{\nabla} \mu_{\mathrm{n}}+\rho \boldsymbol{\nabla} \Phi=\boldsymbol{F}_{\mathrm{L}},
$$

where $\rho=\rho_{\mathrm{p}}+\rho_{\mathrm{e}}+\rho_{\mathrm{n}}$ is the total mass density. This equation can also be written in terms of the pressure gradient, since $\nabla P=n_{\mathrm{p}} \boldsymbol{\nabla} \mu_{\mathrm{p}}+n_{\mathrm{e}} \boldsymbol{\nabla} \mu_{\mathrm{e}}+n_{\mathrm{n}} \boldsymbol{\nabla} \mu_{\mathrm{n}}$. In this case, for a barotropic equation of state, magnetostatic equilibrium now requires that the Lorentz force per unit mass $\boldsymbol{F}_{\mathrm{L}} / \rho$ be a gradient. This leads to the well-known Grad-Shafranov equation, which determines the structure of the magnetic field.

A standard way to obtain the electric field to study the dynamics of a multi-constituent plasma is to consider the appropriate linear combinations of equations (13) and (14) to derive a generalized Ohm's law describing the time variation of the electric current (see e.g. Goedbloed \& Poedts 2004). The same procedure has been applied to neutron stars in different works (Goldreich \& Reisenegger 1992; Shalvbkov \& Urpin 1995; Passamonti et al. 2017) to obtain a general expression for the electric field. Assuming $m_{\mathrm{p}} \gg m_{\mathrm{e}}$, neglecting inertial terms, and dropping gradients which are inconsequential for the induction equation
(8), we get

$$
\boldsymbol{E} \simeq \frac{\boldsymbol{j}}{\sigma_{0}}-\frac{\boldsymbol{v}_{\mathrm{c}}}{c} \times \boldsymbol{B}+\frac{\boldsymbol{j}}{e n_{\mathrm{c}} c} \times \boldsymbol{B} .
$$

Here $\sigma_{0}$ denotes the electrical conductivity in the absence of a magnetic field, and the velocity of the charged fluid is given by

$$
\boldsymbol{v}_{\mathrm{c}}=\frac{\rho_{\mathrm{p}} \boldsymbol{v}_{\mathrm{p}}+\rho_{\mathrm{e}} \boldsymbol{v}_{\mathrm{e}}}{\rho_{\mathrm{c}}} .
$$

We note that the $\boldsymbol{v}_{\mathrm{c}} \times \boldsymbol{B}$ and $\boldsymbol{j} \times \boldsymbol{B}$ terms in the previous equation can be written in a number of different, but equivalent, ways. The three basic unknowns are the three velocities of the constituents, and in principle, any linear combination of them can be used. A natural choice for one of the velocities is the current (or equivalently $\boldsymbol{u}$, the relative velocity between protons and electrons), which gives the last (Hall) term. We have decided to use the charged plasma velocity $\boldsymbol{v}_{\mathrm{c}}$ to describe the second term, usually identified with ambipolar diffusion since it physically represents the advection of magnetic field lines with the charges, which modifies the magnetic field even in the limit when electron and proton velocities are the same (and no currents are present). The third velocity could be, for example, the velocity of neutrons $\boldsymbol{v}_{\mathrm{n}}$, or the hydrodynamical velocity of the fluid

$$
\boldsymbol{v}=\frac{\rho_{\mathrm{p}} \boldsymbol{v}_{\mathrm{p}}+\rho_{\mathrm{e}} \boldsymbol{v}_{\mathrm{e}}+\rho_{\mathrm{n}} \boldsymbol{v}_{\mathrm{n}}}{\rho},
$$

which are usually neglected when the background is assumed to be in equilibrium. We also note that Equation (17) is consistent with the electric field obtained from the electron Euler equation (equation 10), in the limit $m_{\mathrm{e}} \ll m_{\mathrm{p}}$.

\subsection{Long-term evolution of the magnetic field}

A common approach to study the effects of the slow processes is to consider a system in steady state and filter out the short timescale dynamics (e.g. sound and Alfvén waves). This is done by neglecting the inertial terms in equations (13)-(15). However, as noted before, for a barotropic fluid, equation (16) then requires that the Lorentz force per unit mass be a gradient. Even if the fluid is not barotropic, in axisymmetry, the hydrostatic terms cannot balance arbitrary magnetic fields, particularly the $\phi$ component. This would inevitably result in the appearance of accelerations and the activation of fast dynamical flows. Therefore, the configuration of the magnetic field would rapidly change before the Hall and Ohmic processes had time to further influence the evolution.

This problem does not arise in the neutron star crust. Since it is solid, a matter flow cannot be established and any additional force can be balanced by the elastic response of the crust (up to the breaking point). However, in the fluid core, the quasi-static evolution shows serious limitations. Assuming a slow evolution is still a useful compromise to investigate the effects of the Hall drift and Ohmic dissipation, but it remains unclear what the magnetic field geometry will be after the fluid core is relaxed by fast dynamical processes.

In general, allowing for fluid motion and acceleration, and using the equations of continuity (equation 1), the total 
momentum equation can be written as

$$
\begin{aligned}
& \frac{\partial(\rho \boldsymbol{v})}{\partial t}+\boldsymbol{\nabla} \cdot\left(\rho \boldsymbol{v} \boldsymbol{v}+\frac{\rho_{\mathrm{c}} \rho_{\mathrm{n}}}{\rho} \boldsymbol{w} \boldsymbol{w}\right.\left.+\frac{\rho_{\mathrm{e}} \rho_{\mathrm{p}}}{\rho_{\mathrm{c}}} \boldsymbol{u} \boldsymbol{u}\right) \\
&+\nabla P+\rho \boldsymbol{\nabla} \Phi=\boldsymbol{F}_{\mathrm{L}} .
\end{aligned}
$$

where we have defined $\boldsymbol{w}=\boldsymbol{v}_{\mathrm{c}}-\boldsymbol{v}_{\mathrm{n}}$. For stationary flows the partial derivatives $\partial / \partial t$ are neglected, and if one can further argue that the $\boldsymbol{w}$ and $\boldsymbol{u}$ terms are small compared to the $\boldsymbol{v}$ term, then this equation reduces to the one fluid case.

Although an evident improvement over the static assumption, the inclusion of velocities in the stationary regime must be accompanied by a new advective term in the induction equation, which may become dominant. Equation (20) is not easy to solve in the general case. As far as we know, in the literature there are not yet numerical solutions which describe the internal magnetic field of neutron stars with flow motion. Analytical solutions have been presented only for simplified cases (Chandrasekhar 1956; Tsinganos 1981, 1982).

\section{MAGNETIC FIELD EVOLUTION IN A SUPERCONDUCTING NEUTRON STAR CORE}

Protons in the core of a neutron star undergo a phase transition to a type-II superconducting state when the temperature drops below $\lesssim 10^{10} \mathrm{~K}$. The magnetic field then penetrates the core in the form of a dense array of thin fluxtubes. In this section, we consider the dynamical equations by using the Newtonian HVBK formalism 11 for a plasma formed by normal electrons, superconducting protons and superfluid neutrons. These particles are respectively indicated with the letters e, p and n. For simplicity, we neglect the entrainment between nucleons and we omit thermal fluctuations in the superconducting protons and superfluid neutrons, i.e. we are well below the critical temperatures. All protons are therefore in the superconducting state and quasi-particle excitations are absent. We consider a locally neutral system, $n_{\mathrm{p}}=n_{\mathrm{e}} \equiv n_{\mathrm{c}}$.

As in the normal case, the dynamics of this plasma at zero temperature can be described by a system of three Euler and three mass conservation equations. It is possible to choose the Euler and mass conservation equations for each fluid species, or otherwise use a combination of these three equations to describe the motion of the total fluid. The HVBK formalism provides the total conservation equation and describes separately the Euler equations for the superconducting and superfluid particles (Mendell \& Lindblom 1991; Gusakov \& Dommes 2016).

The hydrodynamical system of equations formed by a "normal" and a "superconducting" component are very similar to the normal fluid case detailed in the previous section, with a few remarkable differences. In particular, we note that:

(i) The true Lorentz force $\boldsymbol{F}_{\mathrm{L}}$ is negligible, because, in type II superconductors, Ampère's law connects the

1 We use Newtonian theory in order to have an easier comparison with the formalism of Glampedakis et al. (2011), but our discussion is equally valid in the relativistic limit.
London field $\boldsymbol{b}_{\mathrm{L}}$ to the macroscopic average currents (Glampedakis et al. 2011),

$$
\boldsymbol{\nabla} \times \boldsymbol{b}_{\mathrm{L}}=\frac{4 \pi}{c} \boldsymbol{j} .
$$

and the London field is very weak $\boldsymbol{b}_{\mathrm{L}} \ll \boldsymbol{B}$. For the same reason, at the hydrodynamical averaged scale it is safe to assume that $u \ll v_{\mathrm{p}} \approx v_{\mathrm{e}}$ (i.e. no macroscopic currents).

(ii) However, there is now a new superconducting force, the tension/buoyancy of the fluxoids $\boldsymbol{T}$, which can be written in the following compact form,

$$
\boldsymbol{T}=\frac{\mathcal{C} \times \boldsymbol{B}}{c}
$$

where

$$
\mathcal{C} \equiv \frac{c}{4 \pi} \nabla \times\left(H_{c 1} \hat{\boldsymbol{b}}\right) .
$$

As shown by Dommes \& Gusakov (2017), the vector field $\boldsymbol{T}$ also contains the contribution of the buoyancy force. Equation (23) has the same mathematical form as Ampère's law in the normal case, with the difference that the magnetic induction $\boldsymbol{B}$ is replaced by the vector $H_{c 1} \hat{\boldsymbol{b}}$, where $H_{c 1}$ is the lower critical magnetic field (Tinkham 2004). In type II superconducting neutron stars,

$$
H_{c 1} \approx 10^{15}\left(\frac{n_{\mathrm{p}}}{0.01 \mathrm{fm}^{-3}}\right) \mathrm{G} .
$$

Note that this is equivalent to replacing the macroscopic current $\boldsymbol{j}$ in the Lorentz force by an effective current $\mathcal{C}$.

(iii) The mutual friction terms look different and they have a different physical origin. For example, $\boldsymbol{F}_{\text {pe }}$ describing the mutual friction between the protons and electrons is (Bekarevich \& Khalatnikov 1961; Mendell 1991b)

$$
\boldsymbol{F}_{\mathrm{pe}}=-\left(1+\alpha \rho_{\mathrm{p}}\right) \boldsymbol{T}-\beta \rho_{\mathrm{p}} \hat{\boldsymbol{b}} \times \boldsymbol{T}-\gamma \rho_{\mathrm{p}} \hat{\boldsymbol{b}}\left(\boldsymbol{B} \cdot \frac{\mathcal{C}}{c}\right) .
$$

The third term is normally neglected because its strength is small compared to the other two (Bekarevich \& Khalatnikov 1961). The coefficients $\alpha$ and $\beta$ are related to the dimensionless drag coefficient $R$ (typically $R \sim 10^{-4}$ ) by (Dommes \& Gusakov 2017)

$$
\alpha \rho_{\mathrm{p}}=-\frac{1}{1+R^{2}} \quad \text { and } \quad \beta \rho_{\mathrm{p}}=\frac{R}{1+R^{2}} .
$$

For brevity, we do not discuss the particular forms of the vector fields $\boldsymbol{F}_{\mathrm{en}}$ and $\boldsymbol{F}_{\mathrm{pn}}$, describing mutual friction forces between neutrons and the charged particles, as these are not important for this work. For example, they can describe the electron scattering off the magnetized core of a neutron vortex (Alpar et al. 1984) or the interaction between proton and neutron vortices (see e.g. Sauls 1989; Ruderman et al. 1998).

We now consider the Euler equations. After neglecting inertial terms, and other terms of the order of the weak London field, the equations for the superconducting protons, normal electrons and superfluid neutrons in the laboratory reference frame read

$$
\begin{aligned}
& n_{\mathrm{p}} \boldsymbol{\nabla} \hat{\mu}_{\mathrm{p}}+\rho_{\mathrm{p}} \boldsymbol{\nabla}=\boldsymbol{T}+\boldsymbol{F}_{\mathrm{pe}}+\boldsymbol{F}_{\mathrm{pn}}+e n_{\mathrm{p}}\left(\boldsymbol{E}+\frac{\boldsymbol{v}_{\mathrm{p}}}{c} \times \boldsymbol{B}\right), \\
& n_{\mathrm{e}} \boldsymbol{\nabla} \mu_{\mathrm{e}}+\rho_{\mathrm{e}} \boldsymbol{\nabla} \Phi=-\boldsymbol{F}_{\mathrm{pe}}+\boldsymbol{F}_{\mathrm{en}}-e n_{\mathrm{e}}\left(\boldsymbol{E}+\frac{\boldsymbol{v}_{\mathrm{e}}}{c} \times \boldsymbol{B}\right) \\
& n_{\mathrm{n}} \boldsymbol{\nabla} \hat{\mu}_{\mathrm{n}}+\rho_{\mathrm{n}} \boldsymbol{\nabla} \Phi=-\boldsymbol{F}_{\mathrm{en}}-\boldsymbol{F}_{\mathrm{pn}} .
\end{aligned}
$$


Here, the chemical potentials $\hat{\mu}_{\mathrm{p}}$ and $\hat{\mu}_{\mathrm{n}}$ may also contain contributions due to the magnetic field energy (Glampedakis et al. 2011) and kinetic terms. Equation (28) is equivalent to the electron Euler equation provided in Glampedakis et al. (2011), since the mutual friction force density (25) is exactly the force used in their work and in Graber et al. (2015). In equation (27) we have replaced the force $f_{\mathrm{p}}$ which was used in Gusakov \& Dommes (2016), with the relation $\rho_{\mathrm{p}} n_{\mathrm{p}} f_{\mathrm{p}}=-\boldsymbol{T}-\boldsymbol{F}_{\mathrm{pe}}$.

The sum of equations (27)-(29) gives

$$
n_{c} \boldsymbol{\nabla}\left(\hat{\mu}_{\mathrm{p}}+\mu_{\mathrm{e}}\right)+n_{n} \boldsymbol{\nabla} \hat{\mu}_{\mathrm{n}}+\rho \boldsymbol{\nabla} \Phi=\boldsymbol{T},
$$

which implies that, in hydrostatic equilibrium, and for a barotropic equation of state

$$
\nabla \times\left(\frac{\boldsymbol{T}}{\rho}\right)=0 .
$$

This is the condition for magnetostatic equilibrium in superconducting neutron stars, and has been studied in a number of works (Roberts 1981; Akgün \& Wasserman 2008; Lander 2013; Henriksson \& Wasserman 2013; Lander 2014; Palapanidis et al. 2015).

\subsection{Evolution timescales}

Surprisingly, although the momentum equations given by Glampedakis et al. (2011) and Gusakov \& Dommes (2016) are equivalent, Dommes \& Gusakov (2017) find that their evolution timescales differ by several orders of magnitude from the results of Glampedakis et al. (2011). We now discuss the cause of this discrepancy. In Gusakov \& Dommes (2016) and Dommes \& Gusakov (2017), their approximation for the electric field is derived from the Euler equation of the superconducting protons (equation 27),

$\left.e \boldsymbol{E}\right|_{\mathrm{p}} \equiv-\frac{\boldsymbol{T}+\boldsymbol{F}_{\mathrm{pn}}}{n_{c}}-\left(\frac{e}{c} \boldsymbol{v}_{\mathrm{p}} \times \boldsymbol{B}+\frac{\boldsymbol{F}_{\mathrm{pe}}}{n_{\mathrm{c}}}\right)+\nabla \hat{\mu}_{\mathrm{p}}+m_{\mathrm{p}} \boldsymbol{\nabla} \Phi$,

which, using equation (29), can be written as

$$
\begin{aligned}
\left.e \boldsymbol{E}\right|_{\mathrm{p}} & \equiv-\frac{\boldsymbol{T}}{n_{c}}+\frac{\boldsymbol{F}_{\mathrm{en}}}{n_{c}}-\left(\frac{e}{c} \boldsymbol{v}_{\mathrm{p}} \times \boldsymbol{B}+\frac{\boldsymbol{F}_{\mathrm{pe}}}{n_{\mathrm{c}}}\right) \\
& +\frac{n_{c} \boldsymbol{\nabla} \hat{\mu}_{\mathrm{p}}+n_{n} \boldsymbol{\nabla} \hat{\mu}_{\mathrm{n}}+\left(\rho_{\mathrm{p}}+\rho_{\mathrm{n}}\right) \boldsymbol{\nabla} \Phi}{n_{c}}
\end{aligned}
$$

In Glampedakis et al. (2011), it is determined from the electron equation (28),

$$
\left.e \boldsymbol{E}\right|_{\mathrm{e}} \equiv \frac{\boldsymbol{F}_{\mathrm{en}}}{n_{c}}-\left(\frac{e}{c} \boldsymbol{v}_{\mathrm{e}} \times \boldsymbol{B}+\frac{\boldsymbol{F}_{\mathrm{pe}}}{n_{\mathrm{c}}}\right)-\nabla \mu_{\mathrm{e}}-m_{\mathrm{e}} \boldsymbol{\nabla} \Phi .
$$

In both cases the inertial terms are neglected.

It is evident that both approximations to the electric field are equivalent, because one can use equation (30) to change from one expression to the other. The problem here is that the apparently large contribution of the tension term $\boldsymbol{T} / n_{\mathrm{c}}$, estimated from equation (32), in reality must be considered in combination with the total momentum equation. In fact, from equation (33), we can see that the various gradient terms balance the contribution of the $\boldsymbol{T} / n_{\mathrm{c}}$.

To highlight the issue, let us momentarily proceed by ignoring the gradient terms in equations (32) and (34) as well as $\boldsymbol{F}_{\text {en }}$ and $\boldsymbol{F}_{\text {pn }}$ in comparison with $\boldsymbol{F}_{\text {pe }}$ (see Glampedakis et al. 2011). As noted above, this procedure may be misleading when the total momentum equation is not considered. After using the explicit form of the mutual friction force (25), the electric field approximated by equations (32) and (34) can be written in the following compact form

$\boldsymbol{E}=-\frac{\boldsymbol{v}_{\mathrm{p}}}{c} \times \boldsymbol{B}+\frac{1}{e n_{\mathrm{c}}}\left(\frac{R^{2}}{1+R^{2}}-\chi\right) \boldsymbol{T}+\frac{1}{e n_{\mathrm{c}}} \frac{R}{1+R^{2}} \hat{\boldsymbol{b}} \times \boldsymbol{T}+\ldots$,

where the parameter $\chi$ provides the link between the two cases: $\chi=1$ for equation (32), $\chi=0$ for equation (34). It is important to remark that this parameter appears only in the second term of equation (35), which is proportional to the fluxtube tension. Comparing with the normal matter case where (equation 17)

$$
\boldsymbol{E} \simeq \frac{\boldsymbol{j}}{\sigma_{0}}-\frac{\boldsymbol{v}_{\mathrm{c}}}{c} \times \boldsymbol{B}+\frac{\boldsymbol{F}_{\mathrm{L}}}{e n_{\mathrm{c}}}
$$

we can note three differences: 1) there is no Ohmic dissipation in the superconductor; 2) the non-dissipative Hall term $\left(\propto \boldsymbol{F}_{\mathrm{L}}\right)$ is replaced by a similar term $\left.(\propto \boldsymbol{T}) ; 3\right)$ there is a new term, proportional to $\hat{\boldsymbol{b}} \times \boldsymbol{T}$, which has the same mathematical structure as ambipolar diffusion as defined in Goldreich \& Reisenegger (1992).

From equation (35) and the induction equation, we can extract two timescales describing the long-term evolution of the magnetic field. As shown by Graber et al. (2015) and Dommes \& Gusakov (2017), one can determine a conservative (mathematically, a Hall-like term) and a dissipative timescale. The controversy is about the estimate of the conservative timescale

$$
\tau_{\text {con }}=\frac{e \rho_{p}}{m_{\mathrm{p}} c} \frac{4 \pi L^{2}}{H_{\mathrm{c} 1}}\left(\frac{R^{2}}{1+R^{2}}-\chi\right)^{-1},
$$

with the parameter $L$ denoting a typical length-scale of the system. To recover the result of Graber et al. (2015), we take $\chi=0$, so that $\tau_{\text {con }}=\mathcal{O}\left(R^{2}\right)$, and

$$
\tau_{\text {con }} \approx 1.3 \times 10^{15} L_{6}^{2} \text { yr },
$$

while for Dommes \& Gusakov (2017), $\chi=1, \tau_{\text {con }}=\mathcal{O}(1)$ and

$$
\tau_{\text {con }} \approx 2 \times 10^{8} L_{6}^{2} \text { yr. }
$$

The large difference between the two estimates is apparently caused by the presence of a much larger term $\propto \boldsymbol{T} / n_{\mathrm{c}}$. As pointed out before, from the total momentum equation (30), we can see that the combination of $\boldsymbol{T} / n_{\mathrm{c}}$ with the various gradients present in (33) is an irrotational field. Hence, this term does not lead to any change in the magnetic field after inserting the electric field in the induction equation.

The timescale (38) should be considered as a lower limit, because if the leading term in the electric field is nearly irrotational $\tau_{\text {con }}$ is even longer. To correctly estimate the evolution timescale of the magnetic field, we should first calculate the solenoidal part of the electric field, after combining all terms, but this requires a detailed prescription of the magnetic field geometry and to know the velocity field. For general magnetic fields, if the system is not required to be strictly in magnetostatic equilibrium, the motion of the fluid and inertial terms may not be negligible, and the approximations usually made are questionable. In principle, there can be dynamical readjustments that modify the magnetic field geometry much faster than the slow, secular processes. 


\section{CONCLUSIONS}

We have reconsidered the problem of the magnetic field evolution in a multicomponent plasma, and discussed the approximations normally used in the context of long-term evolution of magnetic fields in neutron stars. The standard approach assumes that hydro-magnetic equilibrium is reached in the fluid core immediately after formation (within tens of rotation/Alfvén timescales). After this first stage, slow processes, such as Ohmic dissipation, Hall drift and ambipolar diffusion, modify the magnetic field on much longer timescales. This approximation is motivated by the multiscale nature of the problem, which makes it extremely difficult to carry out a numerical study of the complete system of MHD equations. However, the assumption of hydromagnetic equilibrium also places a strong constraint on the form of the magnetic force, which must be mostly irrotational (in the absence of inertial terms). Therefore, terms in the electric field proportional to the magnetic force (either the Lorentz force for normal matter, or the fluxoid tension for superconducting matter) must be treated with extreme caution when estimating evolution timescales, as only the solenoidal parts contribute to the induction equation, and these can be many orders of magnitude smaller.

The preceding remark clarifies a recent controversy that appeared in the literature, about the "correct" expression for the induction equation in superconducting neutron stars. This is an important issue, as a disagreement of seven orders of magnitude between estimates of timescales (see Graber et al. 2015 and Dommes \& Gusakov 2017) would lead to very different conclusions about the evolutionary scenario. Our analysis shows that the evolution timescale of Dommes \& Gusakov (2017) is likely an overestimation, and that the one given in Graber et al. (2015) and Elfritz et al. (2016) should be closer to describe the slow evolution of the magnetic field in a neutron star core.

More generally, as the magnetic field evolves, the magnetic force per unit mass will very likely quickly acquire a solenoidal component. In the fluid core, this will inevitably activate fluid motions which, acting on dynamical timescales, would modify the magnetic field faster than the Ohmic and Hall processes (or their equivalent dissipative and non-dissipative processes in superconducting cores). This problem does not arise for the crust of a neutron star, which is instead modeled as a solid ion lattice plus a single electron fluid. The elasticity of the crust can sustain small deviations from equilibrium for any magnetic field of reasonable strength.

A significant improvement in our understanding of magnetic field evolution in neutron star cores would be to construct stationary (but not static) solutions of the momentum and mass conservation equations, which would allow us to obtain the velocity field explicitly, and to incorporate the corresponding advective terms in the electric field and the induction equation. This may significantly alter the secular evolution timescales in neutron star cores.

\section{ACKNOWLEDGEMENTS}

A.P. acknowledges support from the European Union under the Marie Sklodowska Curie Actions Individual Fellowship, grant agreement $\mathrm{n}^{\circ} 656370$. This work is supported in part by the Spanish MINECO grant AYA2015-66899-C2-2-P, and by the New Compstar COST action MP1304.

\section{REFERENCES}

Akgün T., Wasserman I., 2008, MNRAS, 383, 1551

Alpar M. A., Langer S. A., Sauls J. A., 1984, ApJ, 282, 533

Bekarevich I., Khalatnikov I., 1961, Sov. J. Exp. Theor.

Phys, 13, 643

Chandrasekhar S., 1956, ApJ, 124, 232

Dommes V. A., Gusakov M. E., 2017, MNRAS Lett., 467, L115

Elfritz J. G., Pons J. A., Rea N., Glampedakis K., Viganò D., 2016, MNRAS, 456, 4461

Glampedakis K., Andersson N., Samuelsson L., 2011, MNRAS, 410, 805

Goedbloed J. P. H., Poedts S., 2004, Principles of Magnetohydrodynamics. Cambridge University Press

Goldreich P., Reisenegger A., 1992, ApJ, 395, 250

Graber V., Andersson N., Glampedakis K., Lander S. K., 2015, MNRAS, 453, 671

Gusakov M. E., 2016, Phys. Rev. D, 93, 064033

Gusakov M. E., Dommes V. A., 2016, Phys. Rev. D, 94, 083006

Hall H. E., 1960, Advances in Physics, 9, 89

Hall H. E., Vinen W. F., 1956, Proceedings of the Royal Society of London Series A, 238, 215

Henriksson K. T., Wasserman I., 2013, MNRAS, 431, 2986

Khalatnikov I. M., 2000, An Introduction to the Theory of Superfluidity. Westview Press, New York

Lander S. K., 2013, Phys. Rev. Lett., 110, 071101

Lander S. K., 2014, MNRAS, 437, 424

Mendell G., 1991a, ApJ, 380, 515

Mendell G., 1991b, ApJ, 380, 530

Mendell G., Lindblom L., 1991, Annals of Physics, 205, 110

Palapanidis K., Stergioulas N., Lander S. K., 2015, MNRAS, 452, 3246

Passamonti A., Akgün T., Pons J. A., Miralles J. A., 2017, MNRAS, 465, 3416

Roberts P. H., 1981, Q. J. Mech. Appl. Math. XXXIV, 327

Ruderman M., Zhu T., Chen K., 1998, ApJ, 492, 267

Sauls J., 1989, in Ögelman H., van den Heuvel E. P. J., eds, Timing Neutron Stars. Kluwer, Dordrecht

Shalybkov D. A., Urpin V. A., 1995, MNRAS, 273, 643

Tinkham M., 2004, Introduction to superconductivity, 2nd edn.. Dover Publications, New York

Tsinganos K. C., 1981, ApJ, 245, 764

Tsinganos K. C., 1982, ApJ, 252, 775 